\title{
Isolation and Expression Analysis of Brassica rapa WRKY 7
}

\author{
Seon-Seol Kim ${ }^{1}$, Yu Jin Ko ${ }^{1}$, Ji-Young Jang ${ }^{1}$, Theresa Lee ${ }^{1}$, Myung-Ho Lim ${ }^{2}$, Sang-Yeol Park ${ }^{1}$, Shin-Chul \\ Bae $^{1}$, Choong-Hyo Yun ${ }^{1}$, Beom-Seok Park ${ }^{2}$ and Duk-Ju Hwang ${ }^{1 *}$ \\ ${ }^{\prime}$ Department of Molecular Physiology and Biochemistry \\ ${ }^{2}$ Brassica Genomics Team, National Institute of Agricultural Biotechnology (NIAB), RDA, Suwon, Korea \\ (Received on August 11, 2008; Accepted on November 5, 2008)
}

\begin{abstract}
The cDNA clone of Brassica rapa WRKY7 (BrWRKY7) was obtained from EST collection in Brassica genomics team and its DNA sequence was determined. The cDNA clone is 1,037 bp long in nucleotides and encodes an open reading frame of 307 amino acids. Based on a phylogenetic tree, BrWRKY7 belongs to group IId. BrWRKY7 was induced by wound and SA. It was also induced by pathogen attack such as Xanthomonas campestris pv. campestris (Xcc), suggesting that this $B r W R K Y$ may play an essential role in defense response of chinese cabbages.
\end{abstract}

Keywords : Brassica rapa, WRKY transcription factor

Plants have a variety of active defense mechanisms to protect themselves from pathogen infection. Plant defense responses result from the transcriptional activation of a large number of genes upon pathogen infection or treatment with pathogen elicitors (Ruston and Somssich, 1998; Schreiber and Desveaux, 2008; Yang et al., 1997). Most WRKY genes are rapidly induced by pathogens, pathogen elicitors, or salicylic acid (SA) treatment in mostly Arabidopsis and rice (Chen and Chen, 2000; Dong et al., 2003; Eulgem et al., 1999; Ryu et al., 2006). Therefore, it has been implicated that WRKY proteins are major transcriptional regulators in defense signaling (Chen and Chen, 2000, 2002; Chen et al., 2002; Cormack et al., 2002; Deslandes et al., 2002; Du and Chen, 2000; Eulgem et al., 1999; Kim et al., 2000; Liu et al., 2005, 2006; Maleck et al., 2000; Robatzek and Somssich, 2001, 2002; Yoda et al., 2002; Yu et al., 2001). In this study, based on global expression profiling analysis of Brassica rapa genes on $25 \mathrm{~K}$ cDNA oligomer chip, BrWRKY7 was isolated and characterized.

Chinese cabbage (Brassica rapa ssp. pekiness cv. chiibu) was grown in a greenhouse for 3 weeks. Three-week old chinese cabbage seedlings were treated with salicylic acid (SA) at $1 \mathrm{mM}$ or buffer, respectively. Chinese cabbage

\footnotetext{
*Corresponding author.

Phone) +82-31-299-1742, FAX) +82-31-299-1722

E-mail)djhwang@rda.go.kr
}

leaves were harvested at the times indicated in the figures. For bacterial inoculations, a compatible strain of Xcc to Brassica rapa ssp. pekiness cv. chiibu was grown in PSA

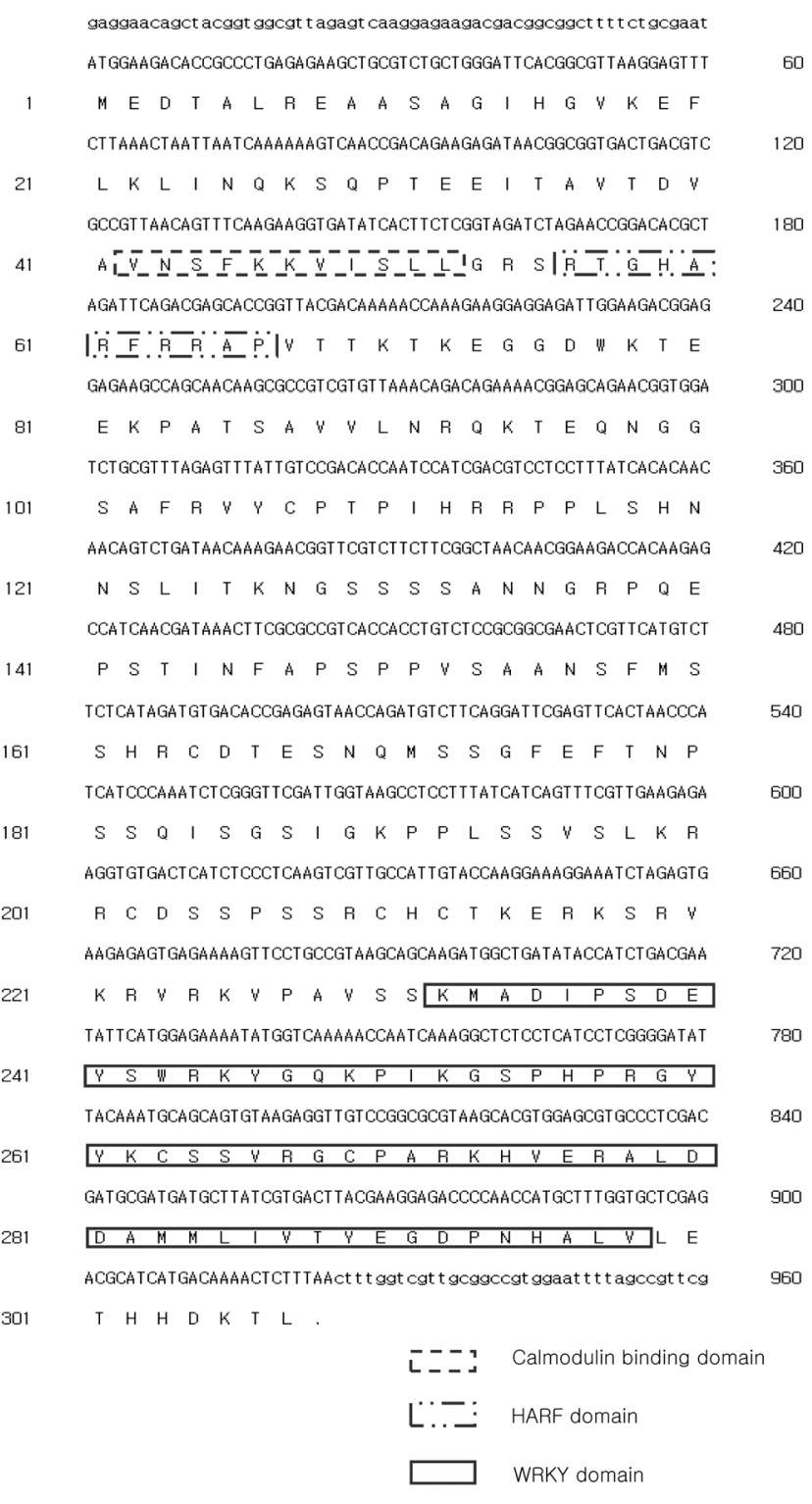

Fig. 1. Nucleotide sequence and deduced amino acids of BrWRKY7. Typical DNA binding domain in the most WRKY proteins was boxed. 
medium (10 g peptone, $10 \mathrm{~g}$ sucrose, $1 \mathrm{~g}$ sodium-glutamate, and $15 \mathrm{~g}$ agar per liter) for 2 days and then resuspended in 1 $\mathrm{mM} \mathrm{MgCl} 2$ to a final $\mathrm{OD}_{600}$ of 0.5 . Inoculumn of Xcc was sprayed on 3-week-old Chinese cabbage seedlings. After inoculations, plants were incubated in a humidity chamber for $24 \mathrm{hr}$. Samples were taken at $24 \mathrm{hr}$. Leaf samples were ground to powder in liquid nitrogen and total RNA was extracted using Trizol reagent according to the manufacturer's instructions (Invitrogen, Carlsbad, CA). RT-PCR analysis using M-MLV RTase (Promega, Madison, WI) was performed with $1 \mu \mathrm{g}$ of total RNA according to manufacturer's instructions and method described before (Lee, J.-H. et al., 2008) Subsequent PCR was performed for 2535 cycles using specific primers 5'-TTCACTAACCCATCATCCCAAATC-3' and 5'-AAACAAGAGTAGGGTCACTTCCAAC-3' for BrWRKY7. PCR product with primers 5'-TGTGAACGATTCCTGCACCT-3' and 5'-GGAAGGATCTGTACGGTAAC-3' for BrActin (In-Ah Lee, MS thesis, 2005) was used as eual loading controls.

The cDNA clone of BrWRKY7 was obtained from the collection of expressed sequence tags (ESTs) in our institute. Its cDNA clone is $1,037 \mathrm{bp}$ long in nucleotides and encodes a protein of 307 amino acids. Its nucleotide sequences and deduced amino acid sequences are shown in Fig. 1. The WRKY domain of BrWRKY7 is underlined. To investigate the function of $B r W R K Y 7$, a phylogenetic tree was constructed based on WRKY domain sequences (60 amino acids) of WRKY proteins as shown in Fig. 2. BrWRKY7 belongs to group IId. Seven members of AtWRKY proteins (AtWRKY7, AtWRKY11, AtWRKY15, AtWRKY17, AtWRKY21, AtWRKY39, and AtWRKY74) belong to group IId (Fig. 2). According to the phylogenetic tree shown in Fig. 2, BrWRKY7 is a Brassica rapa homolog of AtWRKY7. To investigate the percentage of identity of two
WRKY proteins, we aligned amino acid sequences of their full length proteins by ClustalW in DNAstar software Package (Fig. 3). BrWRKY7 showed 76.5\% identity with AtWRKY7 at amino acid level. Eulgem et al. (2000) reported that group IId WRKY proteins contain a calmodulin binding domain and HARF domain. Fig. 3 showed that these two domains are completely conserved in $\mathrm{BrWRKY} 7$ and $A t$ WRKY7, suggesting that BrWRKY7 functions in a similar way with AtWRKY7. However, we cannot exclude the possibility of a different function with AtWRKY7 because 28 amino acids stretch is not found in the Nterminus of BrWRKY7 whereas it is present in AtWRKY7.

Among seven members of group IId $A t$ WRKY proteins the expression of AtWRKY7, AtWRKY11, AtWRKY15, and $A t \mathrm{WRKY} 17$ is strongly induced, whereas the expression of AtWRKY21, AtWRKY39, AtWRKY74 is not induced upon Pseudomonas syringae pv. tomato (Pst) infection (Journot-Catalino et al., 2006). The expression profile of $A t \mathrm{WRKY} 7$, AtWRKY11, and AtWRKY17 are similar whereas the other group II AtWRKYs is dissimilar. AtWRKY7, AtWRKY11, and AtWRKY17 are reported to be negative regulators in defense signaling against $P s t$ infection (Journot-Catalino et al., 2006; Kim et al., 2006). Kim and his colleagues (2006) also demonstrated that AtWRKY7 is a transcriptional repressor and enhances susceptibility to Pst by analyzing T-DNA and RNA silencing wrky7 mutants. Fig. 4 showed the expression pattern of $B r W R K Y 7$ upon pathogen infection. The transcript level of BrWRKY7 was induced at $6 \mathrm{hr}$ post -inoculation (hpi) (Fig. 4) and also induced at $6 \mathrm{hr}$ after treatment with SA (Fig. 4).

In conclusion, we demonstrated that BrWRKY7 was upregulated upon pathogen infection as AtWRKY7 and besides a WRKY domain $B r$ WRKY7 shared two important conserved domains with AtWRKY7. This suggests that

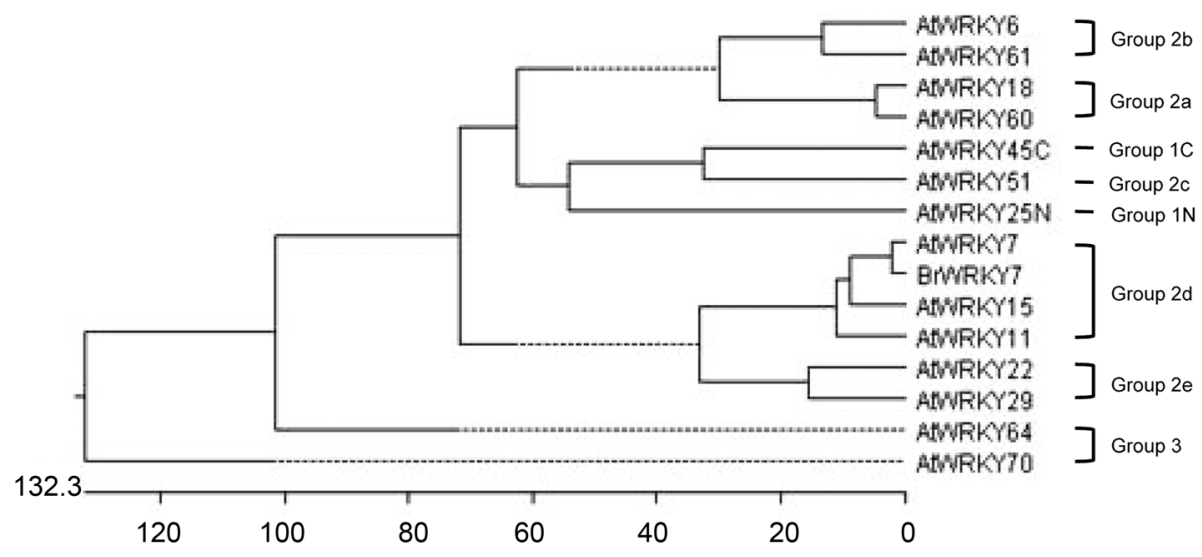

Fig. 2. Phylogenetic tree of BrWRKY7 with other AtWRKY proteins. The phylogenetic tree of BrWRKY7 with other AtWRKY proteins was generated using the neighbor-joining method after alignment of the WRKY domain sequences of AtWRKY6, AtWRKY61, AtWRKY18, AtWRKY60, AtWRKY45C, AtWRKY51, AtWRKY25N, AtWRKY7, AtWRKY15, AtWRKY11, AtWRKY22, AtWRKY29, AtWRKY64, AtWRKY70 with ClustalW. Distance in the tree corresponds to evolutionary distance. 


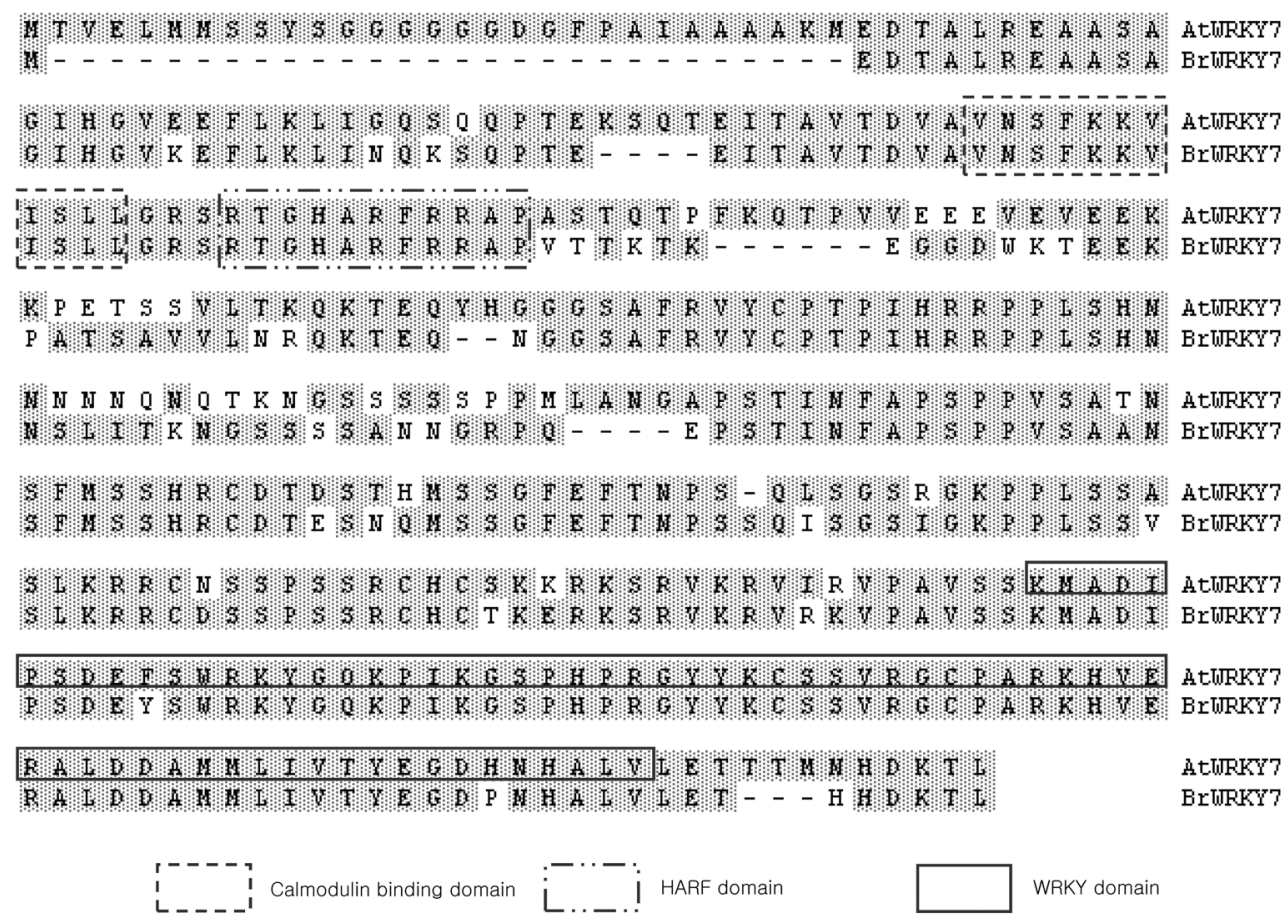

Fig. 3. Comparisions of their amino acid sequences between AtWRKY7 and BrWRKY7. Amino acid sequences of two proteins were aligned by ClustalW in DNAstar software package. Identical amino acids are in boxes.

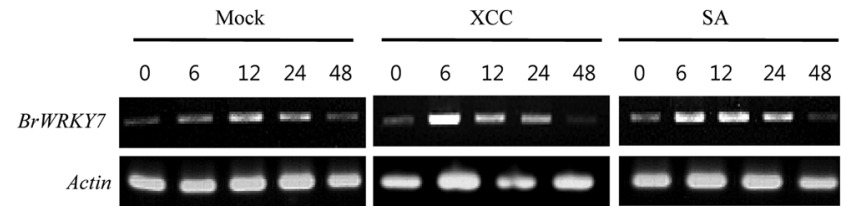

Fig. 4. Expression patterns of BrWRKY 7 in $\mathrm{SA} / X c c$ treated chinese cabbage leaves. Three-week-old chinese cabbage seedlings were infected with $X c c$ and treated with SA. Immediately after treatment of chinese cabbage seedlings, leaves were harvested as a sample at $0 \mathrm{hr}$ and the rest of the samples were taken at 6 , 12,24 , and $48 \mathrm{hr}$. Total RNA was isolated from each sample and RT-PCR was performed using BrWRKY7-specific primer pairs. Transcript levels of BrActin show that equal amounts of RNA were used in the RT-PCR samples.

$B r$ WRKY7 appears to act as a negative regulator in defense signaling in relation to pathogen and SA as $A t$ WRKY7. To verify this hypothesis on the function of $B r$ WRKY7, ectopic expression of $B r W R K Y 7$ should be tried and complementation by over-expression of BrWRKY7 into T-DNA wrky7 mutant should also be tried in near future.

\section{Accession Numbers}

Locus identifiers of the genes from this article are as follows: At1g62300 (WRKY6), At1g18860 (WRKY61), At4g31800 (WRKY18), At2g25000 (WRKY60), At3g01970 (WRKY45), At5g64810 (WRKY51), At2g30250 (WRKY25),
At4g24240 (WRKY7), At2g23320 (WRKY15), At4g31550 (WRKY11), At4g01250 (WRKY22), At4g23550 (WRKY29), At1g66560 (WRKY64), At3g56400 (WRKY70).

\section{Acknowledgements}

This work was supported, in part, by a grant CG3134 from 21st Century Frontier Crop Functional Genomics and grants from the National Institute of Agricultural Biotechnology (NIAB), Rural Development Administration (RDA) to Dr. Duk-Ju Hwang. Dr. Ji Young Jang is the recipient of a postdoctoral fellowship from the Korea Research Foundation.

\section{References}

Chen, C. and Chen, Z. 2002. Potentiation of developmentally regulated plant defense response by AtWRKY18, a pathogeninduced Arabidopsis transcription factor. Plant Physiol. 129:706-716.

Chen, C. and Chen, Z. 2000. Isolation and characterization of two pathogen- and salicylic acid-induced genes encoding WRKY DNA-binding proteins from tobacco. Plant Mol. Biol. 42:387396.

Chen, W., Provart, N. J., Glazebrook, J., Katagiri, F., Chang, H. S., Eulgem, T., Mauch, F., Luan, S., Zou, G., Whitham, S. A., Budworth, P. R., Tao, Y., Xie, Z., Chen, X., Lam, S., Kreps, J. A., Harper, J. F., Si-Ammour, A., Mauch-Mani, B., Heinlein, 
M., Kobayashi, K., Hohn, T., Dangl, J. L., Wang, X. and Zhu, T. 2002. Expression profile matrix of Arabidopsis transcription factor genes suggests their putative functions in response to environmental stresses. Plant Cell 14:559-574.

Cormack, R. S., Eulgem, T., Rushton, P. J., Kochner, P., Hahlbrock, K. and Somssich, I. E. 2002. Leucine zipper-containing WRKY proteins widen the spectrum of immediate early elicitor-induced WRKY transcription factors in parsley. Biochim. Biophys. Acta. 1576:92-100.

Deslandes, L., Olivier, J., Theulieres, F., Hirsch, J., Feng, D. X., Bittner-Eddy, P., Beynon, J. and Marco, Y. 2002. Resistance to Ralstonia solanacearum in Arabidopsis thaliana is conferred by the recessive $R R S 1-R$ gene, a member of a novel family of resistance genes. Proc. Natl. Acad. Sci. USA 99:2404-2409.

Dong, J., Chen, C. and Chen, Z. 2003. Expression profiles of the Arabidopsis WRKY gene superfamily during plant defense response. Plant Mol. Biol. 51:21-37.

$\mathrm{Du}, \mathrm{L}$. and Chen, Z. 2000. Identification of genes encoding receptor-like protein kinases as possible targets of pathogen- and salicylic acid-induced WRKY DNA-binding proteins in Arabidopsis. Plant J. 24:837-847.

Eulgem, T., Rushton, P. J., Schmelzer, E., Hahlbrock, K. and Somssich, I. E. 1999. Early nuclear events in plant defense signaling: Rapid gene activation by WRKY transcription factors. EMBO J. 18:4689-4699.

Journot-cataline, N., Somissich, I. E., Roby, D. and Kroi, T. 2006. The transcription factors WRKY11 andWRKY 17 ant as negative regulators of basal resistance in Arabidopsis thaliana. Plant Cell 18:3289-3302.

Kim, C. Y., Kee, S. H., Park, H. C., Bae, C. G., Cheong, Y. H., Choi, Y. J., Han, C., Lee, S. Y., Lim, C. O. and Cho, M. J. 2000. Identification of rice blast fungal elicitor-responsive genes by differential display analysis. Mol. Plant-Microbe Interact. 13:470-474.

Kim, K. C., Fan, B. and Chen, Z. 2006. Pathogen-induces Arabidopsis WRKY7 is a transcriptional repressor and enhances plant susceptibility to Pseudomonas syringae. Plant Physiol. 142:1180-1192.

Lee, J.-H., Hong, J. B., Hong, S. B., Choi, M.-S., Jeong, K. Y.,
Park, H.-J., Hwang, D.-J., Lee, S., Ra, D. and Heu, S. 2008. Disease-resistant transgenic Arabidopsis carrying the exp $\mathrm{I}$ gene from Pectobacterium carotovorum subsp. carotovorum SL940. Plant Pathol. J. 24:183-190.

Liu, X. Q., Bai, X. Q., Qian, Q., Wang, X. J., Chen, M. S. and Chu, C. C. 2005. OsWRKY03, a rice transcriptional activator that functions in defense signaling pathway upstream of OsNPR1. Cell Res. 15:593-603.

Liu, X. Q., Bai, X. Q., Wang, X. J. and Chu, C. C. 2006. OsWRKY71, a rice transcription factor, is involved in rice defense response. J. Plant Physiol. 164:969-979.

Maleck, K., Levine, A., Eulgem, T., Morgan, A., Jürg, S., Lawton, K. A., Dangl, J. L. and Dietrich, R. A. 2000. The transcriptome of Arabidopsis thaliana during systemic acquired resistance. Natl. Genet. 26:403-409.

Robatzek, S. and Somssich, I. E. 2001. A new member of the Arabidopsis WRKY transcription factor family, $A t$ WRKY6, is associated with both senescence- and defense-related processes. Plant J. 28:123-133.

Rushton, P. J. and Somssich, I. E. 1998. Transcriptional control of plant genes responsive to pathogens. Curr. Opin. Plant Biol. 1:311-315.

Ryu, H. S., Han, M., Lee, S. K., Cho, J. I., Ryoo, N., Heu, S., Lee, Y. H., Bhoo, S. H., Wang, G., Hahn, T. R. and Jeon, J. S. 2006. A comprehensive expression analysis of the WRKY gene superfamily in rice plants during defense response. Plant Cell Rep. 25:836-47.

Schreiber, K. and Desveaux, D. 2008. Message in a bottle: Chemical biology of induced disease resistance in plants. Plant Pathol. J. 24:245-268.

Yang, Y., Shah, J. and Klessig, D. F. 1997. Signal perception and transduction in plant disease responses. Genes Dev. 11:16211639.

Yoda, H., Ogawa, M., Yamaguchi, T., Koizumi, N., Kusano, T. and Sano, H. 2002. Identification of early-responsive genes associated with the hypersensitive response to tobacco mosaic virus and characterization of a WRKY-type transcription factor in tobacco plants. Mol. Genet. Genomics 267:154-161. 